\title{
PSA Screening for Prostate Cancer: Why Saying No is a High-Value Health Care Choice
}

Timothy J. Wilt, MD, MPH, ${ }^{\text {a,b }}$ and Philipp Dahm, MD, MHSc c,d

\begin{abstract}
Enthusiasm for cancer screening and treatment of screen-detected cancer has led to widespread prostate-specific antigen (PSA) screening, a marked increase in prostate cancer incidence, and high use of surgical, radiation, and androgen deprivation treatment for screen-detected disease. This has occurred in advance of a full understanding of the clinical and financial tradeoffs. Although questions remain whether lifetime benefits outweigh harms and costs, data indicate that this balance is not favorable through at least 15 years. This article outlines a conceptual framework for determining the value of screening strategies according to screening and treatment intensity. We describe 4 main cancer screening goals and examine whether PSA screening and treatment achieve these goals and thus provide high-value care. Available evidence demonstrates that PSA screening provides at best a small reduction in prostate cancer mortality, and no reduction in all-cause mortality. High-intensity PSA screening and treatment currently practiced in the United States result in substantial harms and large health care expenditures - it is low-value care. The health importance of prostate cancer and the financial costs to patients and society require improved detection and treatment strategies that produce greater value to patients. We propose lower-intensity, higher-value options. However, until evidence supports a higher-value alternative to current PSA screening strategies, physicians should recommend against PSA screening, policymakers should encourage reduced screening, and most men should say no to the PSA test. (J Natl Compr Canc Netw 2015;13:1566-1574)
\end{abstract}

Few health issues have produced more controversy than prostate-specific antigen (PSA) screening for prostate cancer. Screening and early treatment for screen-detected disease may provide large personal and public health benefits. Prostate cancer is common, potentially deadly, and costly. ${ }^{1}$ In the absence of PSA testing, there are no effective early detection options. Treatment for disease that has spread beyond the prostate does not provide cure. Therefore, public and health professional enthusiasm for cancer screening and treatment of screendetected cancer is understandable. ${ }^{2-4}$ However, it has led to widespread PSA screening, a marked increase in

From aMinneapolis VA Health Care System, Center for Chronic Disease Outcomes Research; bUniversity of Minnesota School of Medicine, Department of Medicine; 'Minneapolis Veterans Affairs Health Care System, Urology Section; and dUniversity of Minnesota School of Medicine, Department of Urology, Minneapolis, Minnesota.

Submitted August 6, 2015; accepted for publication

October 13, 2015.

Dr. Wilt has disclosed that he was a member of the ACCP clinical practice guideline committee for prostate cancer screening.

Dr. Dahm has disclosed that he has no financial interests, arrangements, affiliations, or commercial interests with the manufacturers of any products discussed in this article or their competitors.

Correspondence: Timothy J. Wilt, MD, MPH, 1 Veterans Drive

(111-0), Minneapolis, MN 55417. E-mail: Tim.wilt@va.gov prostate cancer incidence, and high use of surgical, radiation, and androgen deprivation treatment for screendetected disease. This has occurred in advance of a full understanding of the clinical and financial tradeoffs. ${ }^{2,4,5}$

Although questions remain whether PSA screening and early treatment for screen-detected prostate cancer provides lifetime benefits that justify harms and costs (highvalue care), data indicate that this balance is not favorable through at least 15 years. ${ }^{6,7}$ As this article argues, for most men, saying no to PSA screening, as currently practiced in the United States, is an appropriate health care choice. We discuss options that are likely to improve screening and treatment value. However, until evidence supports a higher-value alternative to current PSA screening strategies, physicians should recommend against PSA screening, policymakers should encourage reduced screening, and informed patients who inquire about the test should "choose wisely" by saying, "No, thank you."

\section{Cancer Screening Goals and the Concept of High-Value Screening}

Cancer screening has 4 main goals: (1) reduce cancerspecific mortality, (2) reduce all-cause mortality (extend 
Prostate Cancer Screening: A High-Value Approach

Table 1 High-Value and Low-Value PSA Screening Strategies ${ }^{a}$

\section{High Value: \\ Advised}

Population: Men aged 50-69 y

\section{Low Value:}

Not Advised

Population: Men aged $<50$ or $\geq 70 y$, and men of any age not in good health and with $<10$-y estimated life expectancy

Test: PSA blood test

Strategy: Discuss benefits and harms at least once (more if patient requests) with men who inquire about screening, are in good health, and have $>10$-y estimated life expectancy

Order PSA only if informed man expresses clear preference for screening

Frequency: PSA no more often than 2-4 y

\section{Test: PSA blood test}

Strategy: Order PSA test in men who have not had an informed discussion and not expressed clear preference for testing after discussion

Abbreviation: PSA, prostate-specific antigen.

aThis table provides information for average-risk men who do not have severe competing risk for mortality from another condition. The leastintensive recommended strategies are the minimal ones recommended by the American Cancer Society, the US Preventive Services Task Force, the American College of Physicians, the American Academy of Family Physicians, and the American Urological Association (high value). The strategies that are not recommended represent general agreement among groups and signify low-value screening. The rational for not recommending strategies usually involves an unfavorable tradeoff between benefits and harms, a type of value calculation, but does not include costs. Strategies that are not recommended are more intensive than recommended strategies.

Data from Wilt TJ, Harris RP, Qaseem A; on behalf of the High Value Care Task Force of the American College of Physicians. Screening for Cancer: Advice for High Value Care from the American College of Physicians. Ann Intern Med 2015;162:718-725.

life), (3) decrease cancer-associated morbidity, and (4) do so with acceptable harms and costs. Achieving these goals for any screening strategy is difficult.

For decades, the primary focus of prostate (and other) cancer screening programs was detection and treatment of the greatest number of cancers (high intensity or maximum detection and treatment approach). Screening messages were simple, stating that early detection and treatment saved lives with few adverse consequences. ${ }^{4}$ However, even full implementation of an effective screening program would reduce but not eliminate cancer morbidity and mortality. Furthermore, the medical profession, the lay public, and health system payers are increasingly aware of another side of screening: the problems of harms and costs. This awareness led to recommendations and decisions that incorporate tradeoffs between screening benefits and harms and costs. This implicit consideration of the balance of benefits to harms and costs when making health care decisions is termed a value-based approach. 2,3,7

As with all health care interventions, the value of cancer screening can vary depending on many factors, including screening "intensity" (ie, population screened, frequency screened, test sensitivity). An ideal screening strategy is one that is both effective and implemented at an intensity that optimally balances benefits with harms and costs (ie, produces high-value care). ${ }^{7}$ Strategies that are not effective and those that are less or more intensive than this optimal balance are lower value (Figure 1). Unfortunately, PSA screening strategies currently practiced in the United States are high-intensity and not high-value.

\section{Screening is a Cascade of Events, Rather Than a Single Test}

Screening is the first step in a cascade of events in asymptomatic individuals that almost universally leads to additional diagnostic testing to evaluate positive results. Screening ultimately results in the treatment of most screen-detected cancers (Figure 2). Within a time horizon of 10 years or less, screening does not make these patients better but can make them worse. Thus, the evidentiary burden and threshold of benefits versus harms and costs before recommending PSA screening are higher than for diagnostic and treatment decisions for men with disease signs or symptoms.

\section{Cancer Cases are Heterogeneous}

Optimal screening strategies attempt to find the subsets of cancers with the greatest probability of causing health problems and that are treated more effectively at earlier stages. However, screening preferentially detects the large reservoir of silent, slowerprogressing disease before the development of signs 


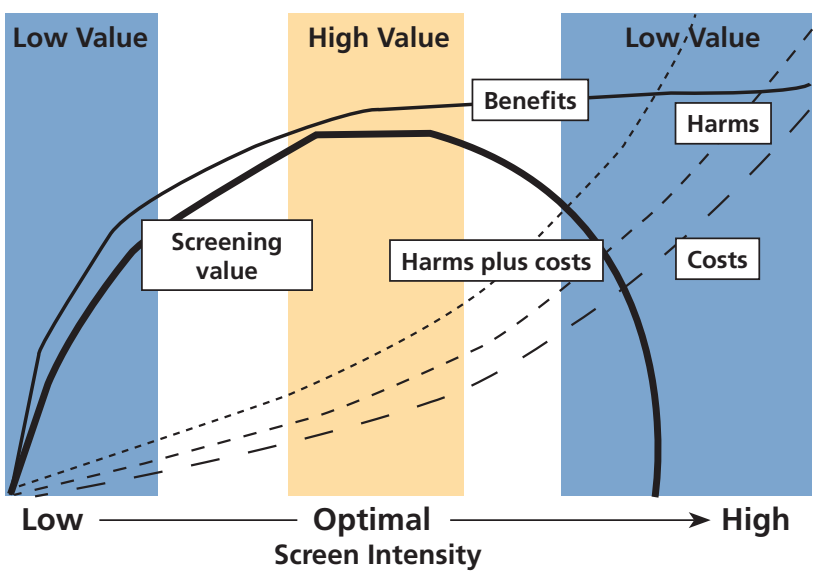

Figure 1 Cancer screening value framework according to screening intensity. The value of cancer screening strategies is linked to screening intensity (population screened, frequency, and sensitivity of test used) and is determined by the balance among benefits (eg, cancer mortality reduction), harms (eg, anxiety from false-positive test results, harms of diagnostic procedures, labeling, overdiagnosis leading to overtreatment), and costs. Low-value care can result from either low benefits or high harms and costs. Low-intensity strategies are initially low-value due to low benefits (left). As intensity increases, benefits increase rapidly with acceptable levels of harms and costs, and value follows an upward trend. Screening strategies provide optimal value when the informed patient or public believes that the balance between benefits and harms or costs is optimal (middle). The top of the value curve is flat because different patients or groups may view different intensities as providing the best balance. Further increases in screening intensity beyond the optimal level lead to slower increases in benefits, with disproportionately rapid increases in harms and costs. Thus, value decreases; higherintensity screening becomes low-value screening (right).

From: Harris RP, Wilt TJ, Qaseem A, on behalf of the High Value Care Task Force of the American College of Physicians. A value framework for cancer screening: advice for high value care from the American College of Physicians. Ann Intern Med 2015;162:713; with permission.

or symptoms (length and lead bias) (Figure 3). Many screen-detected cancers will never cause health problems, even if left untreated (overdiagnosis), yet these individuals are labeled with a cancer diagnosis. As prostate screening intensity has increased, the number of cancer survivors has dramatically increased. This is evidence of increased cancer detection, not proof of benefit. ${ }^{8}$

\section{Patients are Heterogeneous}

Optimal screening strategies focus on persons with sufficient risk for potentially fatal cancer who also have low competing health risks from other causes. However, prostate cancer screening has occurred indiscriminately in younger men at very low disease risk and in older men or those with high competing health risks. Resulting overdiagnosis is due to both detection of slowly progressive cancers (Figure 3) and any cancers detected in patients whose life ends before prostate cancer becomes symptomatic.

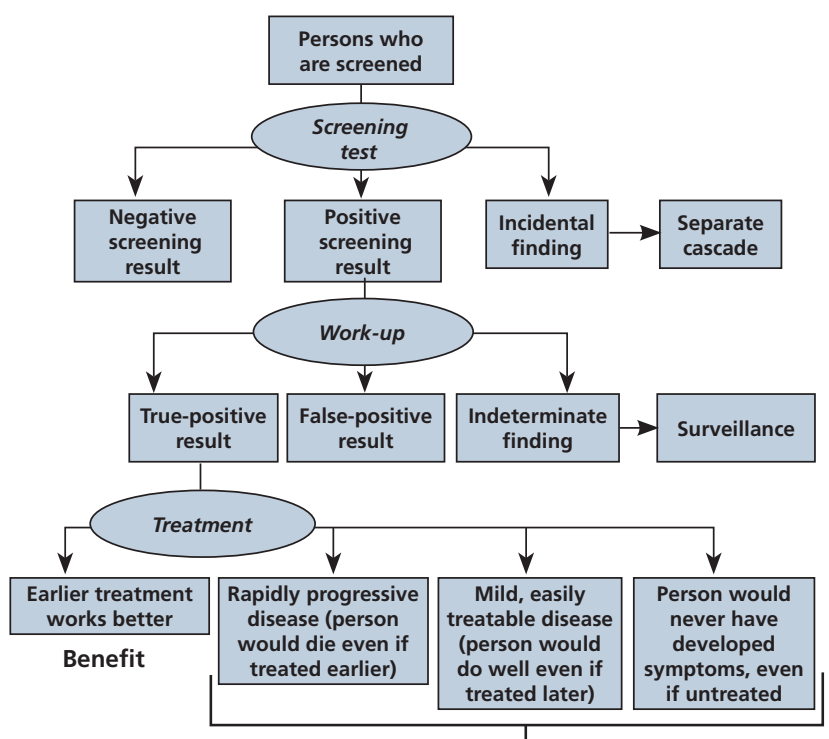

No Benefit

Figure 2 The screening cascade. Screening is not a single test but a cascade of events that can lead to either benefit or harm. The screening test may yield a positive result, a negative result, or an incidental finding (negative for the target condition but with some other abnormality). Patients with an incidental finding are referred for an appropriate workup. Patients with a positive result for the target condition are referred for further diagnostic testing (workup). This leads to a diagnosis in some patients (true-positive result), who are then referred for treatment. However, diagnosis is not the same as benefit. Depending on the need for treatment and the relative effectiveness of earlier (screening detection) versus later (clinical detection) treatment, 4 possible outcomes may occur with treatment after a true-positive result (bottom row, left to right). Earlier treatment leads to benefit, with longer or higher-quality life. The other 3 scenarios provide no benefit, for various reasons. The patient could have rapidly progressive, untreatable disease and would not benefit from earlier detection. Alternatively, the patient could have mild, easily treatable disease and could be treated just as effectively even if the cancer is clinically detected later. Finally, the patient could have either nonprogressive (or slowly progressive) cancer or severe competing mortality risk from another condition and thus would never develop clinically important symptoms from the detected cancer (also known as overdiagnosis). Thus, in 3 of the 4 potential outcomes after screening detection and treatment, there is no benefit. Additionally, every step of the cascade has potential harms, which are immediate, whereas benefits occur only after diagnosis. From Harris RP, Wilt TJ, Qaseem A, on behalf of the High Value Care Task Force of the American College of Physicians. A value framework for cancer screening: advice for high value care from the American College of Physicians. Ann Intern Med 2015;162:714; with permission.

\section{Screening Can Lead to Benefits for Some Cancers} and Patients, but Leads to Harms for Many More Some cancer screening strategies, possibly PSA screening, lead to important benefits for some patients. However, screening leads to significant harms for many more. Any benefit that occurs, although of considerable importance to the individual, does so in the distant future and to a very small number of patients, considering the population screened. In contrast, all are at risk for screening harms. These occur early and often, frequently persist, and can be serious. Because it is difficult to determine which screen-detected cancers will cause future problems, 


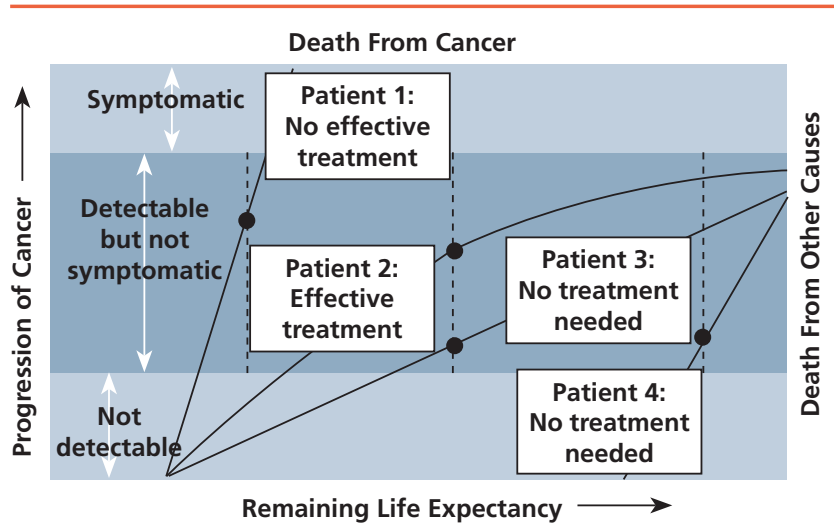

Figure 3 Heterogeneity of cancer cases and patients. Cases of the same type of cancer are heterogeneous in their natural history and response to treatment. Patients are also heterogeneous in their response to treatment and in the presence of serious noncancer health risks. The figure depicts the rate of disease progression for 4 hypothetical patients through 3 zones: not detectable, detectable but not symptomatic, and symptomatic. Screening episodes are represented by the vertical dashed lines, but screening detection (solid circles) occurs only in the second zone (detectable but not symptomatic). For patient 1 , progression is rapid; the cancer may or may not be detected by screening because it spends little time in the detectable but not symptomatic zone. Patient 2 has cancer with an intermediate rate of progression, making it a good target for screening. This cancer has the potential to cause important clinical symptoms (top), and if treatment is more effective in the presymptomatic phase, the treatment bends the natural history curve and the patient benefits from earlier detection. Patient 3 has slowly growing cancer that will not cause symptoms during his or her lifetime. Patient 4 has serious noncancer health risks that decrease life expectancy and prevent benefit from detection of cancer. Because the cancer spends more time in the detectable but not symptomatic zone for patients 3 and 4, it is more likely to be detected by screening than patient 1's cancer; however, the earlier detection is not beneficial because these patients will die of another condition. Patients 3 and 4 are overdiagnosed and usually overtreated, which are both important harms of screening. From Harris RP, Wilt TJ, Qaseem A, on behalf of the High Value Care Task Force of the American College of Physicians. A value framework for cancer screening: advice for high value care from the American College of Physicians. Ann Intern Med 2015;162:712-717

most patients with screen-detected prostate cancers undergo treatment. Treatments have harms that impact quality of life, most notably affecting urinary and sexual functions. Some harms are potentially life-threatening. Treatment cannot provide benefit for individuals who are overdiagnosed; only harm. As screening and treatment intensity increases, incremental benefits are small, but harms and costs increase disproportionately (Figure 1).

\section{Determining Screening Value is Complex, but Possible}

Determining value requires assessing benefits versus harms and costs throughout the screening cascade. This includes the number of persons affected and the clinical importance of each outcome. The balance frequently comes down to many persons experiencing some degree of harm versus a very few experiencing a greater degree of benefit. Clinicians, policymakers, researchers, and patients sometimes differ on the relative importance of these outcomes, and may try summarizing by using "quality-adjusted life years." Although cost is an important factor in determining value, it can be difficult to determine accurately and is rarely incorporated into US clinical guidelines.

\section{Evaluating the Evidence and Applying the Value Framework to PSA Screening for Prostate Cancer}

Before PSA testing, most prostate cancers were detected through a digital rectal examination (DRE) or in men with symptoms of advanced disease, often too late for curative care. PSA screening is advocated as a simple blood test and the best option to reduce disease mortality. Proponents argue that selective use of early treatments is wisely targeted to those most likely to benefit and least likely to be harmed. ${ }^{6}$ What does the current best evidence tell us about the ability of PSA screening to reduce prostate cancer and all-cause mortality and morbidity in a way in which benefits justify harms and costs?

Five large randomized screening trials in more than 300,000 men demonstrate that PSA screening provides at best a small reduction in disease mortality through 15 years $^{5}$ (Figures 4 and 5). There is no reduction in all-cause mortality. ${ }^{9-14}$ Results of the 2 largest studies with the lowest risk of bias indicate that any prostate cancer mortality reduction, if it does exist, is small, likely 1 in 1,000 or less, and confined to men undergoing screening from ages 55 to 69 years and no more frequently than every 2 years. ${ }^{5}$ Follow-up through 13 years in the United States screening study demonstrated no reduction in prostate cancer mortality with 6 years of annual PSA (cutoff of $4.0 \mathrm{ng} / \mathrm{mL}$ ) and 4 years of DRE screening compared with usual care (relative risk $[R R]=1.09$; 95\% CI, 0.87-1.36). ${ }^{5}$ Nearly $90 \%$ of men diagnosed with prostate cancer received active treatment. Results did not differ when excluding individuals without PSA tests before enrollment. PSA screening contamination in the Prostate, Lung, Colorectal, and Ovarian (PLCO) Cancer Screening Trial control group may attenuate differences but would not explain both an increased prostate cancer incidence and increased mortality rate in men assigned to screening, which are concerning. ${ }^{5}$ 
Wilt and Dahm

Of the 5 screening trials, only the European trial reported a reduction in prostate cancer mortality due to screening. ${ }^{9}$ The European trial is essentially pooled results of multiple smaller trials using different patient enrollment, screening, and follow-up criteria. On average, PSA screening occurred every 2 to 7 years, with PSA cutoffs generally ranging from 2.5 to $4.0 \mathrm{ng} / \mathrm{mL}$. Pooled results from 7 sites demonstrate that the relative reduction in cancer mortality was primarily due to large reductions in 2 of the 7 countries reporting (Sweden and the Netherlands) and did not increase after 11 years. ${ }^{9}$ Finland, which enrolled the largest number of participants $(>80,000)$, found no significant cancer mortality reduction. ${ }^{9}$ Additional methodological limitations in the European study include the informed consent process, differential treatments between the study and control groups, and cause-of-death ascertainment, thereby reducing our confidence in their reported estimates of effect. In addition, epidemiologic data are inconclusive. ${ }^{5}$ Some screening trials have suggested a reduction in metastatic disease, but most occurred in cancers detected at the time of diagnosis (stage shift) not after diagnosis (prevention). ${ }^{14,15}$ Results from the United Kingdom-based ProtecT screening trial are expected soon and will be informative. ${ }^{16}$

Recent treatment trials for localized disease demonstrate that reductions as a result of surgery compared with observation in prostate cancer or all-cause mortality and bone metastases are small in absolute terms and mostly limited to men younger than 65 years with palpable tumors; intermediate- or high-risk disease; or PSA levels of $10 \mathrm{ng} / \mathrm{mL}$ or greater. ${ }^{17,18}$ In men with low-risk cancer or who have PSA values of $10 \mathrm{ng} / \mathrm{mL}$ or less and are treated with observation, prostate cancer mortality through 12 years is $5 \%$ or less and does not appear to be substantially reduced by surgery. ${ }^{17}$ Even among men with higherrisk prostate cancer, considerable overdiagnosis and overtreatment exists. For example, at 12 years of follow-up, one study showed that more than $90 \%$ of men with intermediate-risk prostate cancer treated with observation did not die of prostate cancer. ${ }^{17}$ The absolute reduction in prostate cancer mortality due to surgery was small $(<5 \%)$, with the number needed to treat with radical prostatectomy to prevent 1 prostate cancer death equal to 22 . When tumor risk status was reclassified by a central pathologist, there was no mortality reduction (risk difference equal $0.2 \%$ favoring observation). Results for bone metastases were similar. ${ }^{17}$ Based on the current best evidence, radiation therapy does not reduce prostate cancer or all-cause mortality through 15 or more years. ${ }^{19}$ It deserves explicit mention that most men enrolled in the currently reported treatment trials did not have PSA-detected disease. Results from additional ongoing randomized trials in screened men are needed..$^{16}$ However, benefit in men with PSAdetected disease is likely smaller in absolute terms and requires more years to accrue. Thus, PSA screening and treatment for screen-detected prostate cancer fails (or largely fails) the first 3 goals of screening: reduce disease and all-cause mortality and morbidity.

\section{Do Widely Used PSA Screening and Treatment Strategies Yield Acceptable Harms and Costs?}

The current answer is "No." Convincing evidence demonstrates that undergoing a PSA test results in a

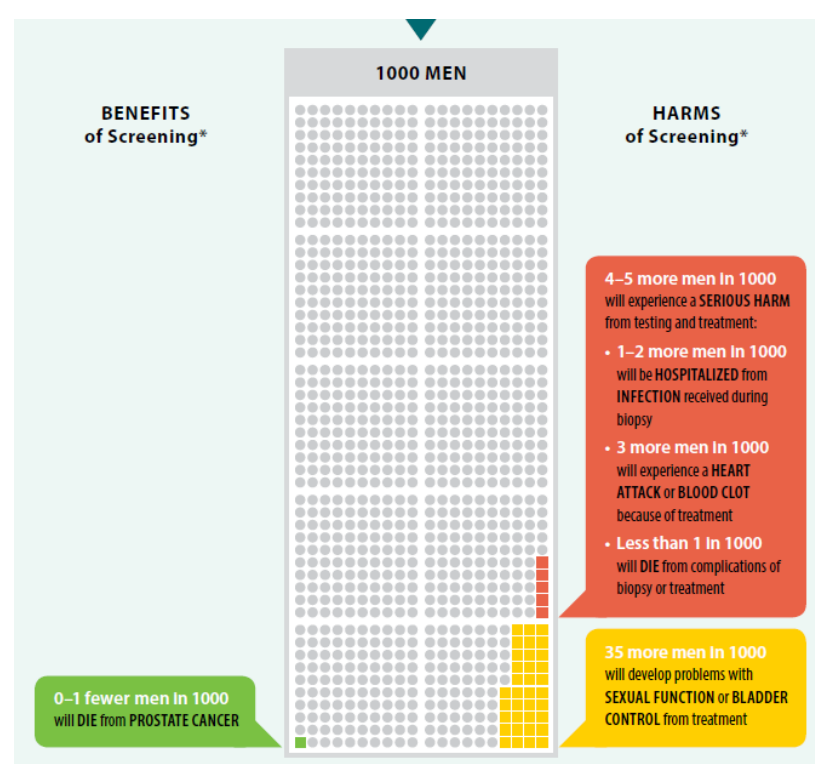

Figure 4 Benefits and harms experienced by men aged 55 to 69 years who are screened for prostate cancer with prostate-specific antigen every 1 to 4 years for 10 years.

From The PSA Test for Prostate Cancer Screening: Why some doctors no longer recommend testing. Available at: http://www.prevention. va.gov/docs/VANCPProstateCancerBr.pdf. Accessed November 9, 2015; with permission.

The information in this graph was obtained from Moyer VA. Screening for Prostate Cancer: US Preventive Services Task Force Recommendation Statement. Ann Intern Med 2012;157:120-134. *The benefits and harms of screening come from treatment and follow-up testing (including biopsy). The information on the harms of treatment is based on the treatments most choose $(60 \%$ chose surgery; $30 \%$ chose radiation; $10 \%$ chose observation). Men who do not seek treatment or who choose observation may experience fewer harms. 
cascade of harmful events and high costs (Figure 4). For 1,000 men undergoing screening every 1 to 4 years and followed up for 10 to 14 years, approximately 1 in 4 will have elevated PSA test results ( $80 \%$ are falsepositive). Most with positive results will undergo at least one set of prostate biopsies - many will have far more than one. Among men undergoing a biopsy, one-third or more will incur harms, including pain, bleeding, and infection. Between 1 and 7 in 100 men receiving biopsies are hospitalized within 30 days, typically for sepsis, many with antibiotic-resistant organisms, ${ }^{5}$ which is an important and increasing problem.

The main screening harm results from detection and subsequent treatment of men diagnosed with prostate cancer. Among 1,000 men screened, 110 will receive a prostate cancer diagnosis. Most of these men will undergo treatment; most will attribute their survival to treatment. However, $16 \%$ to $50 \%$ of men with screen-detected prostate cancer would never experience any sequelae of the prostate cancer they harbor if left untreated..$^{5}$ And treatments have associated harms, including treatmentrelated deaths, bleeding, blood clots, heart attacks, or strokes, and long-term bowel, urinary, or sexual dysfunction. Most screening and treatment harms are of mild to moderate severity but all are far more common than screening's main benefit (avoiding a prostate cancer death, experienced by $0-1$ man of 1,000 screened; Figure 4).

Although we commonly do not consider cost, widespread PSA screening and early intervention results in large costs and is not cost-effective. Annual
2009 Medicare fee-for-service costs for prostate cancer screening and diagnostic tests were $\$ 447$ million, including $\$ 145$ million for men older than 75 years. ${ }^{20}$ Using optimistic assumptions about screening effectiveness and harms, the lifetime cost to prevent one prostate cancer death is $\$ 5,277,308$. The cost per lifeyear saved exceeds $\$ 262,000 .{ }^{21}$ This does not include the economic implications of reduced quality of life as a result of detection and treatment. Incremental cost-effectiveness ratios for PSA screening are not favorable, especially more frequently than every 4 years or outside of men aged 55 to 69 years. ${ }^{22}$ Small life-year gains in quality-adjusted survival are sensitive to optimistic screening benefit estimates and to assumptions of patient values of harms. ${ }^{23}$ Thus, PSA screening and early intervention fail goal number 4 : they do not yield acceptable harms and costs.

\section{Is Overly Intensive Prostate Cancer Screening and Treatment Common?}

Overly intensive low-value prostate cancer screening and treatment are exceedingly common in the United States. One-third of men undergoing PSA testing do not recall being told that the test was ordered. $^{3}$ Most undergoing testing receive annual screening. Even after the publication of the 2012 US Preventive Services Task Force (USPSTF) recommendations, more than one-third of men aged 50 years or older reported receiving PSA testing within the prior 12 months. ${ }^{24}$ Approximately $40 \%$ of men aged 70 years or older reported PSA screen-

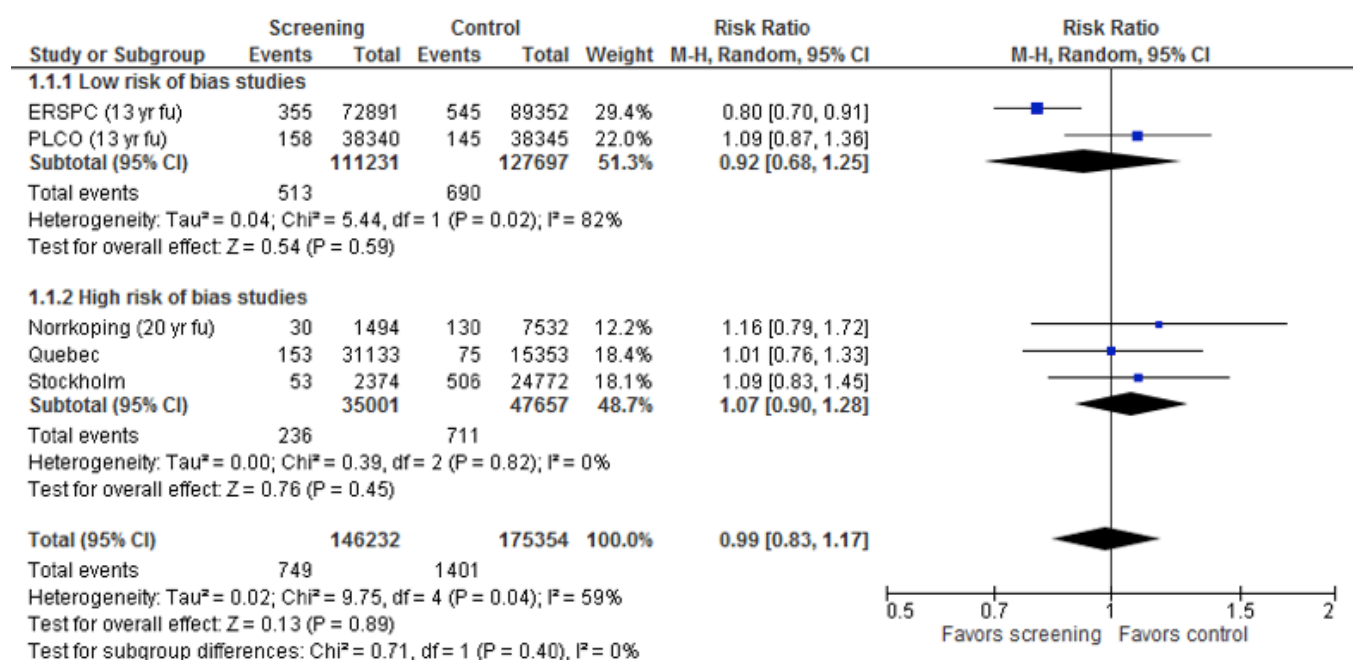

Figure 5 Prostate cancer mortality from prostate-specific antigen-based screening trials. 
Wilt and Dahm

ing despite convincing evidence of harms without benefit. Given the pronounced state-by-state variation, it is unlikely that patient preferences account for this intense screening. Early intervention still is used in most men with low-risk disease..$^{25,26}$ However, cost-effectiveness analyses show that observation or active surveillance for these men results in the greatest quality-adjusted life-years and lowest cost. ${ }^{27}$ Even among men who defer initial local treatment, active surveillance rather than observation is used. Randomized trials have not evaluated the effectiveness of active surveillance, although harms and costs are incurred from periodic biopsies and delayed use of surgery and radiation. For men with higher-risk disease, incidence of early intervention, including surgery, radiation therapy, or androgen deprivation therapy, exceeds $95 \%$ despite convincing evidence of overuse. ${ }^{17}$

\section{What Do Guideline Groups Recommend?}

There is increasing agreement among evidencebased guideline groups about acceptable screening strategies and not recommending overly intensive approaches (Table 1). ${ }^{3}$ All recommend against community-based screening and screening men with life expectancies of 10 years or less. None recommend PSA screening without a discussion of benefits and harms and a patient's expressed clear preference for screening. ${ }^{3}$ All acknowledge that well-informed men may make different screening (and treatment) decisions. This consensus represents a laudable development within recent years. Most guidelines target discussions to men aged 50 to 69 years and recommend testing no more often than every 2 to 4 years in men expressing a clear preference for screening. To date, none incorporate costs into their recommendations, so they do not fully assess value.

Groups vary in the information included; whether clinicians should routinely counsel men (proactive counseling) or only counsel men who inquire about testing (reactive counseling); and what physicians should recommend if patients inquire. A reactive approach and recommending against testing if asked is of higher value., ${ }^{4,2}$ Discussing PSA screening with men who do not inquire about testing takes time and resources away from health care interventions of higher value (opportunity costs). Proactive discussions or recommending testing for other low-value screening strategies (eg, carotid stenosis screening, cervical cancer screening in women $<21$ years of age, lung cancer screening in patients $<55$ years of age or adults who have not smoked for $>15$ years) is not indicated despite the potential prognostic value. Moving forward, we recommend focusing on interventions of high value.

The consensus-based NCCN Clinical Practice Guidelines in Oncology (NCCN Guidelines) for Prostate Cancer Early Detection use a maximal cancer detection framework for men who "opt to participate in an early detection program (after receiving the appropriate counseling on the pros and cons)" (to view the most recent version of these guidelines, visit NCCN. org). ${ }^{29} \mathrm{NCCN}$ recommends informed testing beginning at age 45 years and repeating at 1- to 2-year intervals (through age 75 years) for men who have a PSA value of $1.0 \mathrm{ng} / \mathrm{mL}$ or greater and at 2- to 4-year intervals for men with PSA value less than $1 \mathrm{ng} / \mathrm{mL}$. Screening is recommended in "very select patients" older than 75 years. ${ }^{29}$ NCCN Guidelines do acknowledge the harms and costs due screening, including overdiagnosis and overtreatment. They discourage use of surgery or radiation therapy for most men with low-risk disease. However, compared with strategies recommended by other groups, NCCN Guidelines are high-intensity and low-value (Figure 1). Among men aged 45 to 49 years, PSA testing based on NCCN recommendations would classify approximately one-quarter of those screened as having a PSA above their threshold recommended for frequent follow-up PSA testing. However, no randomized trials have demonstrated a mortality reduction in this age group. Modelling studies suggest that any benefit is at best extremely small $(<1$ in 100,000 men). ${ }^{30}$ Compared with no screening or less-intensive screening, NCCN recommendations for men aged 50 years or older using PSA thresholds of greater than 3.0 $\mathrm{ng} / \mathrm{mL}$ would lead to, at most, very small incremental benefits, with disproportionate increases in costs and harms, especially from prostate biopsies, overdiagnosis, and overtreatment. Meanwhile, for men with PSA values exceeding $3.0 \mathrm{ng} / \mathrm{mL}, \mathrm{NCCN}$ recommends a repeat PSA and, if still elevated, options other than immediate ultrasound-guided biopsies, including monitoring for and/or use of certain biomarkers. This, if widely implemented in men undergoing screening, would be a higher-value strategy than subjecting all men with PSA values exceeding $3.0 \mathrm{ng} / \mathrm{mL}$ to routine prostate biopsies, because it would reduce harms of frequent biopsies 
as well as overdiagnosis and overtreatment, while potentially improving the specificity for the detection of high-grade cancer.

\section{How Can We Improve Prostate Cancer Screening Value?}

Changing screening and treatment beliefs and practices to reduce unnecessary, ineffective (or marginally effective), harmful, and costly health care (lowvalue care) is hard. However, our patients look for evidence-based guidance to help them sort through contradicting opinions. ${ }^{31}$ Our challenge and responsibility is to be the reliable, trusted information source that ensures our patients can make well-informed decisions incorporating best evidence with individual preferences. Well-informed men make PSA screening decisions consistent with lowerintensity recommendations. ${ }^{32,33}$ Knowing this, clinicians and policymakers can improve screening value and ensure patient-centered cost-conscious care by reducing intensity in several ways:

- Target PSA screening discussions to men aged 50-69 years who are in good health $(\geq 10$-year life expectancy) and who inquire about testing

- Use a value framework to describe the limited potential benefits and substantial harms and costs of prostate cancer screening and treatment

- Clinicians should recommend against PSA testing, as they do with other low-value tests and procedures, if men ask their physician for a recommendation

- Physicians should support the PSA testing decisions of well-informed patients

- Screen well-informed individuals who still express a clear personal preference for testing, but

- Widen screening intervals to every 2-4 years

- Increase PSA thresholds defining abnormality (eg, 6-10 ng/mL)

- Discontinue screening in men with low PSA values $(\mathrm{eg},<2.0 \mathrm{ng} / \mathrm{mL})^{34,35}$

- Discontinue screening in men who no longer request testing, develop life-limiting comorbidities, or reach age 70 years

- Develop "overuse" performance measures to reward physicians and health systems for not ordering PSA tests in men

- Without an informed discussion

- Aged $<50$ years

- With a limited life expectancy due to age ( $\geq 70$ years) or life-limiting comorbidities
- Rename low-risk prostate cancer to more accurately describe its indolent nature (eg, prostate lesion of low malignant potential)

- Enhance use of observation rather than active surveillance, surgery, radiation, or androgen deprivation therapy for nearly all men with low-risk disease and increase implementation of observation or active surveillance in men with higher-risk disease, especially those with estimated life expectancies $<10-15$ years $^{36}$

- Rigorously evaluate the effectiveness of noninvasive diagnostic and monitoring methods, such as multiparametric MRI, to reduce harms and costs of active surveillance while ensuring that individuals with higher-risk disease who may benefit from treatment receive it

- Identify more-effective and less-harmful treatments for men with a high prostate cancer mortality risk

These lower intensity, higher-value strategies would reduce diagnostic and treatment harms with no difference in all-cause mortality and little to no change in disease morbidity and mortality. ${ }^{1-5}$ They would reduce costs. Such strategies and future evidence about benefits, harms, and costs, as well as information about US practice patterns, could turn low-value strategies into a high-value approach.

\section{Conclusions}

PSA screening as currently practiced in the United States is low-value health care because it provides little to no reduction in prostate cancer morbidity or mortality, does not decrease all-cause mortality, and results in substantial diagnostic and treatment harms and large health care expenditures. The health importance of prostate cancer and the financial costs to patients and society require improved detection and treatment strategies that produce greater value to patients. Until empirical evidence becomes available to support a higher-value alternative to current PSA screening strategies, men and their health care providers can make a high-value health care choice by saying no to the PSA test.

\section{References}

1. Siegel RL, Miller KD, Jemal A. Cancer statistics, 2015. CA Cancer J Clin 2015;65:5-29.

2. Harris RP, Wilt TJ, Qaseem A; on behalf of the High Value Care Task Force of the American College of Physicians. A value framework for cancer screening: advice for high value care from the American College of Physicians. Ann Intern Med 2015;162:712-717. 
3. Wilt TJ, Harris RP, Qaseem A; on behalf of the High Value Care Task Force of the American College of Physicians. Screening for cancer: advice for high value care from the American College of Physicians. Ann Intern Med 2015;162:718-725.

4. Wilt TJ, Partin MR. Screening: simple messages...sometimes. Arch Intern Med 2011;171:2046-2048.

5. Moyer VA. Screening for prostate cancer: US Preventive Services Task Force recommendation statement. Ann Intern Med 2012;157:120-123.

6. Wilt TJ Scardino PT, Carlsson SV, Basch E. Prostate-specific antigen screening in prostate cancer: perspectives on the evidence. J Natl Cancer Inst 2014;106:1-6.

7. Owens DK, Qaseem A, Chou R, Shekelle P; for the Clinical Guidelines Committee of the American College of Physicians. Cost-conscious health care: concepts for clinicians to evaluate the benefits, harms, and costs of medical interventions. Ann Intern Med 2011;154:174-180.

8. Welch HG, Schwartz LM, Woloshin S. Overdiagnosed. Making People Sick in the Pursuit of Health. Boston: Beacon Press; 2011.

9. Schröder FH, Hugosson J, Roobol MJ, et al. Screening and prostate cancer mortality: results of the European Randomised Study of Screening for Prostate Cancer (ERSPC) at 13 years of follow-up Lancet 2014:384:20272035.

10. Ilic D, Dahm P. Prostate cancer screening in Europe. Lancet 2015;385:1506.

11. Ilic D, Neuberger MM, Djulbegovic M, Dahm P. Screening for prostate cancer. Cochrane Database Syst Rev 2013;1:CD004720.

12. Black WC, Haggstrom DA, Welch HG. All-cause mortality in randomized trials of cancer screening. J Natl Cancer Inst 2002;94:167-173.

13. Dubben HH. Trials of prostate-cancer screening are not worthwhile. Lancet Oncol 2009;10:294-298.

14. Basch E, Oliver TK, Vickers A, et al. Screening for prostate cancer with prostate-specific antigen testing: American Society of Clinical Oncology provisional clinical opinion. J Clin Oncol 2012;30:3020-3025.

15. Carter HB, Albertsen PC, Barry MJ, et al. Early detection of prostate cancer: AUA guideline. Available at: http://www.auanet.org/education/ guidelines/prostate-cancer-detection.cfm. Accessed August 5, 2015.

16. Donovan J, Mills N, Smith M, et al. Quality improvement report: improving design and conduct of randomized trials by embedding them in qualitative research: ProtecT (prostate testing for cancer and treatment) study. BMJ 2002;325:766-770.

17. Wilt TJ, Brawer $M K$, Jones $\mathrm{KM}$, et al. Radical prostatectomy versus observation for early prostate cancer. N Engl J Med 2012;367:203-213.

18. Bill-Axelson A, Holmberg I, Garmo H, et al. Radical prostatectomy versus watchful waiting in localized prostate cancer. N Engl J Med 2014;370:932940.

19. Widmark A, Tomic R, Modig J, et al. Prospective randomized trial comparing external beam radiotherapy versus watchful waiting in early prostate cancer (T1b-T2, pN0, grade 1-2, M0). Presented at the 53rd Annual ASTRO Meeting; October 26, 2011; Miami Beach, Florida.
20. Ma X, Wang R, Long JB, et al. The cost implications of prostate cancer screening in the medicare population. Cancer 2014;120:96-102.

21. Shteynshlyuger A, Andriole GL. Cost-effectiveness of prostate specific antigen screening in the United States: extrapolating from the European study of screening for prostate cancer. J Urol 2011;185:828-832.

22. Pataky R, Gulati R, Etzioni R, et al. Is prostate cancer screening costeffective? A microsimulation model of prostate-specific antigen-based screening for British Columbia, Canada. Int J Cancer 2014;135:939-947.

23. Heijnsdijk EA, Wever EM, Auvinen A, et al. Quality-of-life effects of prostate-specific antigen screening. N Engl J Med 2012;367:595-605.

24. Sammon JD, Pucheril D, Diaz M, et al. Contemporary nationwide patterns of self-reported prostate-specific antigen screening. JAMA Intern Med 2014;174:1839-1841.

25. Cooperberg MR, Carroll PR. Trends in management for patients with localized prostate cancer. JAMA 2015;314:80-81.

26. Maurice MJ, Abouassaly R, Kim SP, Zhu H. Contemporary nationwide patterns of active surveillance use for prostate cancer. JAMA Intern Med 2015;175:1569-1571.

27. Hayes JH, Ollendorf DA, Pearson, SD, et al. Observation versus initial treatment for men with localized, low-risk prostate cancer: a costeffectiveness analysis. Ann Intern Med 2013;158:853-860.

28. Prasad V. It's time to stop screening for prostate cancer. JAMA Intern Med 2014;174:1841-1842.

29. Carroll PR, Parsons JK, Andriole G, et al. NCCN Clinical Practice Guidelines in Oncology: Prostate Cancer Early Detection. Version 2.2015. Available at: NCCN.org. Accessed July 30, 2015.

30. Howard K, Barratt A, Mann GJ, et al. A model of prostate-specific antigen screening outcomes for low- to high-risk men: information to support informed choices. Arch Intern Med 2009;169:1603.

31. Welch HG. Making the call. JAMA 2011;306:2649-2650.

32. Partin MR, Nelson D, Radosevich D. Randomized trial examining the effect of two prostate cancer screening educational interventions on patient knowledge, preferences and behaviors. J Gen Intern Med 2004;19:835-842.

33. Lillie SE, Partin MR, Rice K, et al. The effects of shared decision making on cancer screening-a systematic review. Washington, DC: Department of Veterans Affairs (US); 2014.

34. Carlsson S, Assel M, Sjoberg D, et al. Influence of blood prostate specific antigen levels at age 60 on benefits and harms of prostate cancer screening: population based cohort study. BMJ 2014;348:g2296.

35. Wilt TJ, Dahm P. PSA screening for prostate cancer. BMJ 2014;348:g2559.

36. Ganz PA, Barry JM, Burke W, et al. National Institutes of Health state-of the-science conference: role of active surveillance in the management of men with localized prostate cancer. Ann Intern Med 2012;156:591-595. 